\title{
A potentially novel overlapping gene in the genomes of Israeli acute paralysis virus and its relatives Niv Sabath*, Nicholas Price and Dan Graur
}

Address: Department of Biology and Biochemistry, University of Houston, Houston, TX 77204, USA

Email: Niv Sabath* - nsabath@uh.edu; Nicholas Price - price4890@gmail.com; Dan Graur - dgraur@uh.edu

* Corresponding author

Published: 17 September 2009

Virology Journal 2009, 6:144 doi:10.1186/1743-422X-6-144

This article is available from: http://www.virologyj.com/content/6/I/144

(C) 2009 Sabath et al; licensee BioMed Central Ltd.

This is an Open Access article distributed under the terms of the Creative Commons Attribution License (http://creativecommons.org/licenses/by/2.0), which permits unrestricted use, distribution, and reproduction in any medium, provided the original work is properly cited.
Received: 2 July 2009

Accepted: 17 September 2009

\begin{abstract}
The Israeli acute paralysis virus (IAPV) is a honeybee-infecting virus that was found to be associated with colony collapse disorder. The IAPV genome contains two genes encoding a structural and a nonstructural polyprotein. We applied a recently developed method for the estimation of selection in overlapping genes to detect purifying selection and, hence, functionality. We provide evolutionary evidence for the existence of a functional overlapping gene, which is translated in the + I reading frame of the structural polyprotein gene. Conserved orthologs of this putative gene, which we provisionally call pog (predicted overlapping gene), were also found in the genomes of a monophyletic clade of dicistroviruses that includes IAPV, acute bee paralysis virus, Kashmir bee virus, and Solenopsis invicta (red imported fire ant) virus I.
\end{abstract}

\section{Background}

Colony collapse disorder (CCD) is a syndrome characterized by the mass disappearance of honeybees from hives [1]. CCD imperils a global resource estimated at approximately $\$ 200$ billion [2]. For example, it has been estimated that up to $35 \%$ of hives in the US may have been affected [3]. Many culprits have been suggested as causal factors of CCD, among them fungal, bacterial, and protozoan diseases, external and internal parasites, in-hive chemicals, agricultural insecticides, genetically modified crops, climatic factors, changed cultural practices, and the spread of cellular phones [1]. The Israeli acute paralysis virus (IAPV), a positive-strand RNA virus belonging to the family Dicistroviridae, was found to be strongly correlated with CCD [4]. It was first isolated in Israel [5], but was later found to have a worldwide distribution $[4,6,7]$.

The genome of IAPV contains two long open reading frames (ORFs) separated by an intergenic region. The 5'
ORF encodes a structural polyprotein; the 3' ORF encodes a non-structural polyprotein [5]. The non-structural polyprotein contains several signature sequences for helicase, protease, and RNA-dependent RNA polymerase [5]. The structural polyprotein, which is located downstream of the non-structural polyprotein, encodes two (and possibly more) capsid proteins.

Overlapping genes are easily missed by annotation programs [8], as evidenced by the fact that several overlapping genes were only detected by using the signatures of purifying selection [9-13]. Here, we apply a recently developed method for the detection of selection in overlapping reading frames [14] to the genome of IAPV and its relatives.

\section{Results and Discussion}

In the fourteen completely sequenced dicistroviral genomes (Table 1), we identified 43 same-strand overlap- 
Table I: A list of completely sequenced dicistroviruses used in this study

\begin{tabular}{|c|c|}
\hline Name & Accession number \\
\hline Israel acute paralysis virus (IAPV) & GenBank:NC 009025 \\
\hline Acute bee paralysis virus (ABPV) & GenBank:NC 002548 \\
\hline Kashmir bee virus (KBV) & GenBank:NC 004807 \\
\hline Solenopsis invicta virus (SINV-I) & GenBank:NC 006559 \\
\hline Black queen cell virus (BQCV) & GenBank:NC 003784 \\
\hline Cricket paralysis virus (CrPV) & GenBank:NC 003924 \\
\hline Homalodisca coagulata virus-I (HoCV-I) & GenBank:NC 008029 \\
\hline Drosophila $\mathrm{C}$ virus (DCV) & GenBank:NC 001834 \\
\hline Aphid lethal paralysis virus (ALPV) & GenBank:NC 004365 \\
\hline Himetobi $\mathrm{P}$ virus ( $\mathrm{HiPV})$ & GenBank:NC 003782 \\
\hline Taura syndrome virus (TSV) & GenBank: $\overline{N C \quad 003005}$ \\
\hline Plautia stali intestine virus (PSIV) & GenBank:NC 003779 \\
\hline Triatoma virus $(\mathrm{TrV})$ & GenBank:NC 003783 \\
\hline Rhopalosiphum padi virus (RhPV) & GenBank: $\overline{N C \quad 001874}$ \\
\hline
\end{tabular}

ping ORFs of lengths equal or greater than 60 codons on the positive strand. Ten overlapping ORFs were found in concordant genomic locations in two or more genomes. The concordant overlapping ORFs were assigned to three orthologous clusters (Table 2). The overlapping ORFs in all three clusters are phase- 1 overlaps, i.e., shifted by one nucleotide relative to the reading-frames of the known polyprotein genes. Two of the orthologous clusters overlap the gene encoding the nonstructural polyprotein and one overlaps the reading frame of the structural polyprotein. (In appendix 1, we present the results concerning the overlapping ORFs on the negative strand. We note, however, that dicistroviruses are not known to be ambisense [15].)

We identified a strong signature of purifying selection in cluster A that contains overlapping ORFs from four genomes: IAPV, Acute bee paralysis virus (ABPV), Kashmir bee virus (KBV), and Solenopsis invicta virus 1 (SINV-1)

Table 2: Clusters of orthologous overlapping ORFs on the positive strand

\begin{tabular}{lcccc}
\hline Cluster & Virus & Start of ORF & End of ORF & $\begin{array}{c}\text { Length } \\
\text { (nucleotides) }\end{array}$ \\
\hline \multirow{2}{*}{ A } & IAPV & 6589 & 6900 & 312 \\
& ABPV & 6513 & 6815 & 303 \\
& KBV & 6601 & 6909 & 309 \\
& SINV-I & 4382 & 4798 & 417 \\
\hline \multirow{2}{*}{$B$} & ABPV & 5958 & 6227 & 270 \\
& KBV & 5974 & 6243 & 270 \\
\hline \multirow{2}{*}{ C } & CrPV & 2396 & 2614 & 219 \\
& DCV & 2216 & 2602 & 387 \\
& HoCV-I & 2377 & 2574 & 198 \\
& PSIV & 2333 & 2527 & 195
\end{tabular}

[16-18]. This ORF overlaps the 5' end of the structural polyprotein gene (Figure 1A). The detection of purifying selection is based on a method for the simultaneous estimation of selection intensities in overlapping genes [14]. To ascertain that each overlapping ORF is indeed subject to selection, we used the likelihood ratio test for two hierarchical models. In model 1, we assume no selection on the overlapping ORF. In model 2, the overlapping ORF is assumed to be under selection. If model 2 fits the data significantly better than model $1(p<0.05)$, then the overlapping ORF is predicted to be under selection and is most probably functional. The signature of selection was identified for the ORFs in the three bee viruses (IAPV, ABPV, and KBV). The protein product of the orthologous ORF in SINV-1 could not be tested for selection because the amino acid sequence identity between the ORF from SINV-1 and the ORFs from the three bee viruses (Table 3 ) is lower than the range of sequence identities for which the method can be applied (65-95\%).

An additional indication for selection on these ORFs was obtained by comparing the degrees of conservation of the hypothetical protein sequences of the overlapping ORFs against the protein sequences of the known genes (structural and nonstructural polyproteins, Table 3 ). The degree of amino-acid conservation and, hence, sequence identity between orthologous protein-coding genes is influenced ceteris paribus by the intensity of purifying selection. If both overlapping genes are under similar strengths of selection, the amino-acid sequence identity of one pair of homologous genes would be similar to that of the overlapping pair. On the other hand, if a functional gene overlaps a non-functional ORF, the amino-acid identity between the hypothetical protein sequences of the nonfunctional ORFs would be much lower than that between the two homologous overlapping functional genes. We found that the degree of amino-acid conservation of the overlapping sequence identity between pairs of overlapping ORFs in cluster A is only slightly lower than that of the known gene (maximum of $12 \%$ difference between IAPV and SINV-1 in cluster A, Table 3). In contrast, the amino-acid sequence identity between ORF pairs in clusters $\mathrm{B}$ and $\mathrm{C}$ is much lower than that between the pairs of known genes (maximum of $44 \%$ difference between $\mathrm{CrPV}$ and DCV in cluster C, Table 3 ).

The signature of purifying selection on the ORFs in cluster A suggests that they may encode functional proteins. We provisionally term this gene pog (predicted overlapping gene). In Figure 1, we show that pog is found in the genomes of four viruses that constitute a monophyletic clade, but not in any other dicistrovirid genome (Figure 1A). Its phylogenetic distribution suggests that pog originated before the divergence of SINV-1 from the three bee viruses. The phylogenetic distributions of the ORFs in 
Table 3: Sequence conservation in comparisons of known orthologous proteins and orthologous products of overlapping ORFs.

\begin{tabular}{lcccc}
\hline Cluster & \multicolumn{2}{c}{ Genome pair } & Identity of known proteins (\%) & Identity of hypothetical product of overlapping ORFs (\%) \\
\hline \multirow{2}{*}{ A } & IAPV & ABPV & 80.2 & 74.8 \\
& ABPV & KBV & 79.3 & 75.6 \\
& IAPV & KBV & 77.4 & 72.5 \\
& IAPV & SINV-I & 42.7 & 30.3 \\
& ABPV & SINV-I & 41.6 & 32.6 \\
& KBV & SINV-I & 36.3 & 29.4 \\
\hline \multirow{2}{*}{$B$} & KBV & ABPV & 87.7 & 52.3 \\
\hline \multirow{2}{*}{ C } & CrPV & DCV & 80.3 & 36.1 \\
& HoCV-I & PSIV & 64.3 & 40.0 \\
& DCV & HoCV-I & 56.4 & 28.8 \\
& CrPV & HoCV-I & 48.0 & 31.7 \\
& DCV & PSIV & 44.2 & 36.4 \\
& CrPV & PSIV & 35.7 & 25.0 \\
\end{tabular}

clusters B and C (Figure 1B) are patchy. This patchiness is an additional indication that the overlapping ORFs in clusters $\mathrm{B}$ and $\mathrm{C}$ are spurious, i.e., non-functional.

An examination of the DNA alignment of pog (Figures 2) reveals a conservation of the first potential start codon (ATG or CTG) in the +1 reading frame in three out of the four viral genomes (IAPV, ABPV, and SINV-1). As seen in Figure 3, this conservation cannot be explained by constraints on the overlapping polyprotein, in which the corresponding site is variable and encodes different amino acids (His, Asn, and Pro, in IAPV, ABPV, and SINV-1, respectively). We note, however, that we did not find a conserved Kozak consensus sequence [19] upstream of the potential initiation site. This situation is similar to that described in [13].

A protein motif search resulted in several matches, all with a weak score. Two patterns were found in all four proteins: (1) a signature of rhodopsin-like GPCRs (G protein-coupled receptors), and (2) a protein kinase $C$ phosphorylation site (Figure 3 ). Prediction of the secondary structures [20] suggests that the proteins contain two conserved helix domains, separated by 3-5 residues (except for SINV1 , in which one long domain is predicted), at the C-terminus (Figure 3). A search for transmembrane topology [21] indicates that the longer helix may be a transmembranal segment (Figure 3). Although viruses often use GPCRs to exploit the host immune system through molecular mimicry [22-25], the lengths of the proteins encoded by pog are shorter than the average virus-encoded GPCR. Therefore, these proteins may have a different function.

\section{Conclusion}

In this note, we provide evolutionary evidence (purifying selection) for the existence of a functional overlapping gene, pog, in the genomes of IAPV, ABPV, KBV, and SINV1. To our knowledge, this putative gene, whose coding region overlaps the structural polyprotein, has not been described in the literature before.

\section{Methods}

\section{Sequence Data, Processing, and Analysis}

Fourteen completely sequenced dicistrovirid genomes were obtained from NCBI (Table 1). Each genome was scanned for the presence of overlapping ORFs. We used BLASTP [26] with the protein sequences of the known genes to identify matches of orthologous overlapping ORFs (E value $<10^{-6}$ ). Matching overlapping ORFs were assigned into clusters. Within each cluster, we aligned the amino-acid orthologs by using the sequences of the known genes as references. If alignment length of the overlapping sequence exceeded 60 amino-acids, and if the amino-acid sequence identity among the hypothetical genes within a cluster was higher than $65 \%$, we tested for selection on the hypothetical gene (see below).

We aligned the protein sequences of the two polyproteins with CLUSTAW [27] as implemented in the MEGA package [28]. Alignment quality was confirmed using HoT [29]. We reconstructed two phylogenetic trees (one for each polyprotein) by applying the neighbor joining method [30], as implemented in the MEGA package [28]. Trees were rooted by the mid-point rooting method [31] and confidence of each branch was estimated by bootstrap with 1000 replications.

\section{Detection of Selection in Overlapping Genes}

We used the method of Sabath et al. [14] for the simultaneous estimation of selection intensities in overlapping genes. This method uses a maximum-likelihood framework to fit a Markov model of codon substitution to data 
a

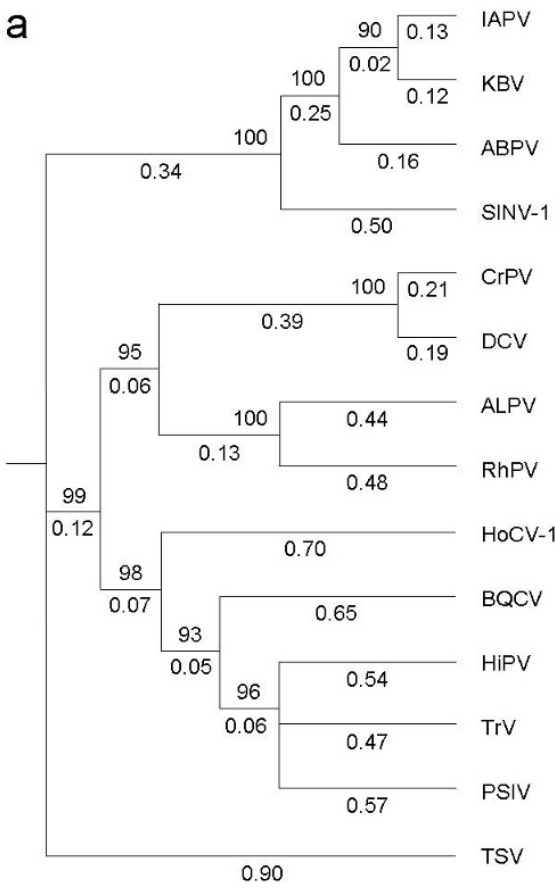

b

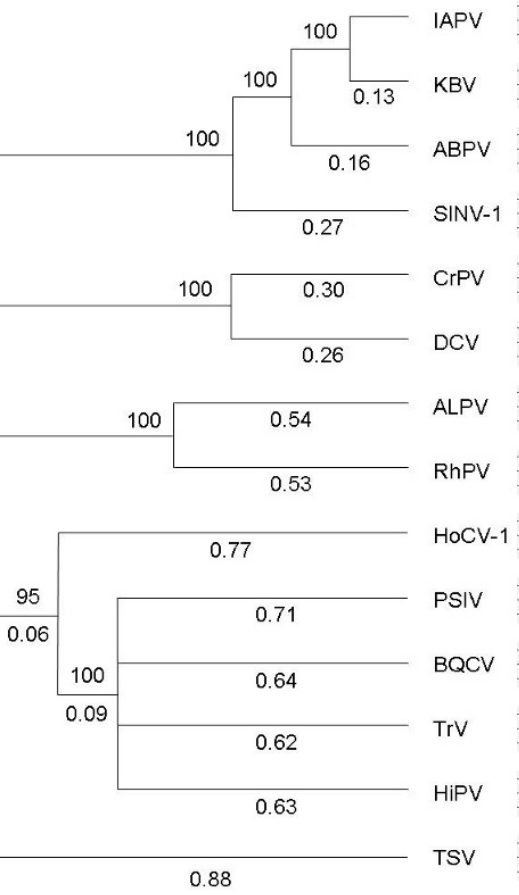

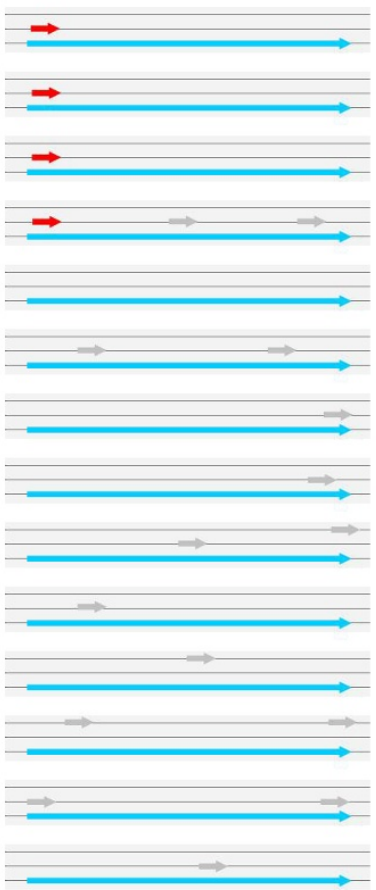
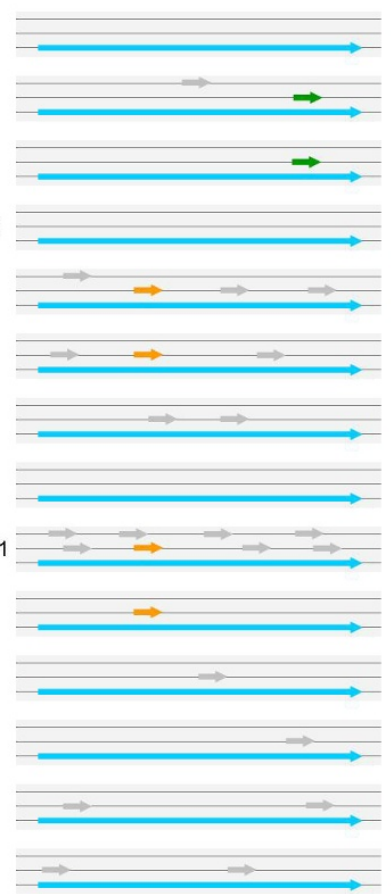

Figure I

Phylogenetic trees and schematic representation of the dicistrovirid genomes (a. structural polyprotein; b. non-structural polyprotein). Trees were inferred using the neighbor joining method [30] and rooted by the mid-point rooting method [3I]. Numbers above and below the branches are bootstrap values ( 1000 replications) and branch lengths (amino-acid substitutions per site), respectively. Phylogenetic analyses were conducted with MEGA [28]. The approximate locations and sizes of the known genes (blue), overlapping hypothetical genes (red, green, and orange), and singlet ORFs (gray) are noted in the three reading frames. 


\begin{tabular}{|c|c|c|c|c|c|c|c|c|c|c|c|c|c|c|c|c|c|c|c|c|}
\hline & 1 & 2 & 3 & 4 & 5 & 6 & 7 & 8 & 9 & 10 & 11 & 12 & 13 & 14 & 15 & 16 & 17 & 18 & 19 & 20 \\
\hline IAPV & gaa & cag & ctg & tac & $\operatorname{tgg}$ & gca & gtt & aca & gca & gtc & gta & $\operatorname{tgg}$ & taa & $\mathrm{cac}$ & atg & cgg & cgt & tcc & gaa & ata \\
\hline $\mathrm{BPV}$ & gaa & cag & cta & tat & $\operatorname{tgg}$ & gta & gtt & gta & gca & gtt & gta & ttc & aaa & tga & atg & cag & cgt & tcc & gaa & 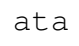 \\
\hline BV & aaa & $\mathrm{ccg}$ & cta & tat & cgg & gta & gct & ata & gca & gtc & gga & tag & taa & tat & atc & $\operatorname{cgg}$ & cgt & ttc & gaa & 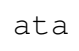 \\
\hline SINV-1 & tag & cag & tca & gga & tgt & cat & tct & ggc & gtt & $\mathrm{Ccg}$ & aaa & tac & $\mathrm{Cca}$ & aac & ctg & ctc & aat & caa & $\mathrm{aca}$ & \\
\hline & 21 & 22 & 23 & 24 & 25 & 26 & 27 & 28 & 29 & 30 & 31 & 32 & 33 & 34 & 25 & 36 & 3 & 38 & 39 & \\
\hline IAPV & $\mathrm{cca}$ & $\operatorname{tgc}$ & ctg & $\operatorname{gcg}$ & att & cac & aac & aag & aaa & gca & ata & ctc & $\mathrm{Cca}$ & $\operatorname{acg}$ & tac & $\mathrm{aca}$ & ata & $\mathrm{cgg}$ & aac & \\
\hline ABPV & tca & tac & ctg & $\mathrm{ccg}$ & atc & --- & --- & aag & aaa & caa & ata & ctt & $\mathrm{Cca}$ & $\operatorname{acg}$ & t & ata & ata & $\mathrm{cgC}$ & aac & \\
\hline KBV & $\mathrm{ca}$ & tac & ctg & ctg & ata & --- & $\mathrm{acc}$ & aag & aaa & $\mathrm{acg}$ & att & cta & $\mathrm{CCa}$ & atg & & ata & $\mathrm{aca}$ & cga & C & \\
\hline$N V-1$ & yd & alc & 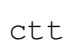 & & aya & yd & $a$ & $\operatorname{cg} 9$ & & Cad & $c$ & y & tt & & & & at & 99 & & \\
\hline
\end{tabular}

\section{Figure 2}

Codon alignment of the 5 ' overlap region between the structural polyprotein and the hypothetical gene. The alignment is shown in the reading frame of the hypothetical gene. The annotated initiation site of the polyproteins is underlined. The first potential initiation site (AUG or CUG) of the hypothetical genes is marked in red. The last stop codon at the +I reading frames is marked in green.

\begin{tabular}{|c|c|c|c|}
\hline \multicolumn{2}{|l|}{ IAPV } & GTAVLGSYSSRMVTHAAFRNTMP GDSQQESNTPNVHNTELASSTSENSVETQEITTFHDV & 60 \\
\hline \multicolumn{2}{|l|}{$\mathrm{ABPV}$} & GTAILGSCSSCIQMNAAFRNI IPADQ--ETNTSNVHNTQLASTSEENSVETEQITTEHDV & \\
\hline \multicolumn{2}{|l|}{ KBV } & ETAISGSYSSRIVIYPAFRNTIPADN-QENDSTNVHNTKLASTSAENAIEKEQITTEHDV & \\
\hline \multicolumn{2}{|l|}{ SINV-1 } & IAVRMSFWRSEIPKPAQSNNANTFETKTATTSASHAQSELSETTPENSLTRQELTVFHDV & \\
\hline IAPV & +1 & EQLYWAVTAVVW *HMRRSE IP CLA I HNKKAI LP TYT IRNSLRP LVKTRLRP KKSQP FMMW & \\
\hline ABPV & +1 & EQLYWVVVAVVFK *MQRSEISYLP I - -KKQI LPTYI IRNSRRP LKKTQLKRNKSPP FMMW & \\
\hline KBV & +1 & KPLYRVAIAVG * *YIRRFEIPYLLI - TKKTI LPMYI TRNSRRP QRRMP LRRNKSPP FMMW & \\
\hline SINV-1 & +1 & *QSGCHSGVPKYPNLLNQTMR I LLRRKRQQP LLP THNRNLARRPQKIP LP DKNSQF SMML & \\
\hline IAPV & & ETPNRIDTPMAQDTSSARNMDDTHS I IQF LQRPVLIDNIEI IAGTTADANKP LSRYV--- & \\
\hline ABPV & & ETPNRINTPMAQDTSSARSMDDTHS I IQF LQRPVLIDH IEVIAGSTADDNKP LNRYV--- & \\
\hline KBV & & ETPNRIDTPMAQDTSSARSMDDTHS I IQF LQRPVLIDNIEIVAGTTADNNTALSRYV--- & \\
\hline SINV-1 & & EQPRVALP IAPQT TSSLAKLDSTAT IVDF LSRTVVLDQFELVQGESNDNHKP LNAATFKD & \\
\hline IAPV & +1 & KLQIGS IPPWLRILHRLGTWMIRTVLFSFYSAPFSLTTLRSLLEQRPMQTNP LADM* --- & \\
\hline ABPV & +1 & KLQIGS IPPWLKTLHRLGAWMIRTVLF SFYNAPYSLTTLRSLLDQQQMI TNP S IDM* --- & \\
\hline KBV & +1 & KLQI GS IPPWLRILHRLGAWMIRTVLFSFYNAP F SLTTLRLLQEQLP I TTQHSVDM* --- & \\
\hline SINV-1 & +1 & NNLASLFQLLRKRLALLLSLILQRQLWIFFLELLSS INSS LFKVNQT I TTNP LTQQLLKT & \\
\hline re 3 & & $\begin{array}{l}\text { annotated initiation site of the polyproteins is marked in blue. The first potential initiation } \\
\text { edicted by MEMSAT [2I] are marked in blue. Conserved protein kinase } C \text { phosphorylation }\end{array}$ & \\
\hline
\end{tabular}


from two aligned homologous overlapping sequences. To predict functionality of an ORF that overlaps a known gene, we modified an existing approach for predicting functionality in non-overlapping genes [32]. Given two aligned orthologous overlapping sequences, we estimate the likelihood of two hierarchical models. In model 1, there is no selection on the ORF. In model 2, the ORF is assumed to be under selection. The likelihood-ratio test is used to test whether model 2 fits the data significantly better than model 1, in which case, the ORF is predicted to be under selection and most probably functional.

\section{Motifs}

We looked for motifs within the inferred protein sequences encoded by the overlapping ORF by using the motif search server http://motif.genome.jp/ and the MyHits server http://hits.isb-sib.ch/cgi-bin/PFSCAN with the following motif databases: PRINTS [33], PROSITE [34], and Pfam [35]. We used PSIPRED [20] to predict secondary structure, and MEMSAT [21] to predict transmembrane protein topology.

\section{Competing interests}

The authors declare that they have no competing interests.

\section{Authors' contributions}

NS carried out the analysis and wrote the draft manuscript. NP performed the motif search. DG and NP contributed to the interpretation of the results and the final version.

All authors have read and approved the manuscript.

\section{Appendix I \\ Overlapping ORFs on the negative strand}

In the fourteen completely sequenced dicistroviruse genomes (Table 1), we identified 240 overlapping ORFs of length equal or greater than 60 codons on the negative strand. Of the 240 ORFs, 113 were found in concordant genomic locations in two or more genomes. The concordant overlapping ORFs were assigned into 29 clusters (Additional file 1). There are 9, 1, and 19 clusters in phase 0,1 , and 2, respectively. The cluster size ranges from 2 to 9. In two clusters, 5 and 10 , both in phase 2 , there is a weak signature of selection. However, this signature seems to be a false positive, which was driven by the unique structure of opposite-strand phase-2 overlap (Additional file 2). In this structure, codon positions one and two of one gene match codon positions two and one of the overlapping gene. This structure leads to a situation where most changes are either synonymous or nonsynonymous in both overlapping genes and occasionally, to false signal of purifying selection on the overlapping ORF. In addition, one of the clusters (cluster 10) does not constitute a monophyletic clade, and is, therefore, unlikely to be func- tional. We therefore conclude that dicistroviruses most probably do not encode proteins on the negative strand.

\section{Additional material}

\section{Additional file 1}

Clusters of orthologous overlapping ORFs on the negative strands of dicistrovirid genomes.

Click here for file

[http://www.biomedcentral.com/content/supplementary/1743422X-6-144-S1.DOC]

\section{Additional file 2}

The corresponding codon positions of overlapping genes in oppositestrand phase-2. First and second codon positions, in which $5 \%$ and $0 \%$ of the changes are synonymous, are marked in red. Third codon positions, in which $\sim 70 \%$ of the changes are synonymous, are marked in blue. Click here for file

[http://www.biomedcentral.com/content/supplementary/1743422X-6-144-S2.PPT]

\section{Acknowledgements}

We thank Dr. Ilan Sela and an anonymous reviewer for their comments. This work was supported in part by US National Library of Medicine Grant LMOI0009-0I to Dan Graur and Giddy Landan and by the Small Grants Program of the University of Houston.

\section{References}

I. Oldroyd BP: What's killing American honey bees? PLoS Biol 2007, 5:el68.

2. Gallai N, Salles J-M, Settele J, Vaissière BE: Economic valuation of the vulnerability of world agriculture confronted with pollinator decline. Ecological Economics 2009, 68:8I0-82I

3. van Engelsdorp D, Hayes J Jr, Underwood RM, Pettis J: A survey of honey bee colony losses in the U.S., fall 2007 to spring 2008. PLOS ONE 2008, 3:e407I.

4. Cox-Foster DL, Conlan S, Holmes EC, Palacios G, Evans JD, Moran NA, Quan PL, Briese T, Hornig M, Geiser DM, et al.: A metagenomic survey of microbes in honey bee colony collapse disorder. Science 2007, 3 I 8:283-287.

5. Maori E, Lavi S, Mozes-Koch R, Gantman Y, Peretz Y, Edelbaum O, Tanne E, Sela I: Isolation and characterization of Israeli acute paralysis virus, a dicistrovirus affecting honeybees in Israel: evidence for diversity due to intra- and inter-species recombination. J Gen Virol 2007, 88:3428-3438.

6. Blanchard P, Schurr F, Celle O, Cougoule N, Drajnudel P, Thiery R, Faucon JP, Ribiere M: First detection of Israeli acute paralysis virus (IAPV) in France, a dicistrovirus affecting honeybees (Apis mellifera). J Invertebr Pathol 2008, 99:348-350.

7. Palacios G, Hui J, Quan PL, Kalkstein A, Honkavuori KS, Bussetti AV, Conlan S, Evans J, Chen YP, vanEngelsdorp D, et al.: Genetic analysis of Israel acute paralysis virus: distinct clusters are circulating in the United States. J Virol 2008, 82:6209-62 I7.

8. Chen W, Calvo PA, Malide D, Gibbs J, Schubert U, Bacik I, Basta S, O'Neill R, Schickli J, Palese $P$, et al.: A novel influenza A virus mitochondrial protein that induces cell death. Nat Med 200I, 7:1306-13/2

9. Chung BY, Miller WA, Atkins JF, Firth AE: An overlapping essential gene in the Potyviridae. Proc Natl Acad Sci USA 2008, 105:5897-5902

10. Firth $A E$ : Bioinformatic analysis suggests that the Orbivirus VP6 cistron encodes an overlapping gene. Virol J 2008, 5:48.

II. Firth $A E$, Atkins JF: Bioinformatic analysis suggests that the Cypovirus I major core protein cistron harbours an overlapping gene. Virol J 2008, 5:62. 
12. Firth $A E$, Atkins JF: Bioinformatic analysis suggests that a conserved ORF in the waikaviruses encodes an overlapping gene. Arch Virol 2008, 153:1379-1383.

13. Firth $A E$, Atkins JF: Analysis of the coding potential of the partially overlapping 3' ORF in segment 5 of the plant fijiviruses. Virol J 2009, 6:32.

14. Sabath N, Landan G, Graur D: A method for the simultaneous estimation of selection intensities in overlapping genes. PLOS ONE 2008, 3:e3996.

15. Nguyen M, Haenni AL: Expression strategies of ambisense viruses. Virus Res 2003, 93:I4I-I50.

16. de Miranda JR, Drebot M, Tyler S, Shen M, Cameron CE, Stoltz DB, Camazine SM: Complete nucleotide sequence of Kashmir bee virus and comparison with acute bee paralysis virus. J Gen Virol 2004, 85:2263-2270.

17. Govan VA, Leat N, Allsopp M, Davison S: Analysis of the complete genome sequence of acute bee paralysis virus shows that it belongs to the novel group of insect-infecting RNA viruses. Virology 2000, 277:457-463.

18. Valles SM, Strong CA, Dang PM, Hunter WB, Pereira RM, Oi DH, Shapiro AM, Williams DF: A picorna-like virus from the red imported fire ant, Solenopsis invicta: initial discovery, genome sequence, and characterization. Virology 2004, 328: $15 \mid-157$.

19. Kozak M: Comparison of initiation of protein synthesis in procaryotes, eucaryotes, and organelles. Microbiol Rev 1983 , 47: $1-45$.

20. McGuffin LJ, Bryson K, Jones DT: The PSIPRED protein structure prediction server. Bioinformatics 2000, 16:404-405.

21 . Jones DT: Improving the accuracy of transmembrane protein topology prediction using evolutionary information. Bioinformatics 2007, 23:538-544.

22. Murphy PM: Viral exploitation and subversion of the immune system through chemokine mimicry. Nat Immunol 200I, 2:116-122

23. Lalani AS, McFadden G: Evasion and exploitation of chemokines by viruses. Cytokine Growth Factor Rev 1999, 10:219-233.

24. McLysaght A, Baldi PF, Gaut BS: Extensive gene gain associated with adaptive evolution of poxviruses. Proc Natl Acad Sci USA 2003, 100:15655-15660.

25. Hughes AL, Friedman R: Genome-wide survey for genes horizontally transferred from cellular organisms to baculoviruses. Mol Biol Evol 2003, 20:979-987.

26. Altschul SF, Gish W, Miller W, Myers EW, Lipman DJ: Basic local alignment search tool. I Mol Biol 1990, 21 5:403-4I0.

27. Thompson JD, Gibson TJ, Higgins DG: Multiple sequence alignment using ClustalW and ClustalX. Curr Protoc Bioinformatics 2002, Chapter 2(Unit 2):3.

28. Kumar S, Nei M, Dudley J, Tamura K: MEGA: a biologist-centric software for evolutionary analysis of DNA and protein sequences. Brief Bioinform 2008, 9:299-306.

29. Landan G, Graur D: Heads or tails: a simple reliability check for multiple sequence alignments. Mol Biol Evol 2007, 24: I380-। 383

30. Saitou N, Nei M: The neighbor-joining method: a new method for reconstructing phylogenetic trees. Mol Biol Evol 1987, 4:406-425.

3I. Farris JS: Estimating phylogenetic trees from distance matrices. Am Nat 1972, 106:645-668.

32. Nekrutenko A, Makova KD, Li WH: The $\mathbf{K}(\mathbf{A}) / \mathbf{K}(\mathbf{S})$ ratio test for assessing the protein-coding potential of genomic regions: an empirical and simulation study. Genome Res 2002, 12:198-202

33. Attwood TK, Blythe MJ, Flower DR, Gaulton A, Mabey JE, Maudling N, McGregor L, Mitchell AL, Moulton G, Paine K, Scordis P: PRINTS and PRINTS-S shed light on protein ancestry. Nucleic Acids Res 2002, 30:239-241.

34. Hulo N, Bairoch A, Bulliard V, Cerutti L, De Castro E, LangendijkGenevaux PS, Pagni M, Sigrist CJ: The PROSITE database. Nucleic Acids Res 2006, 34:D227-230.

35. Finn RD, Tate J, Mistry J, Coggill PC, Sammut SJ, Hotz HR, Ceric G Forslund K, Eddy SR, Sonnhammer EL, Bateman A: The Pfam protein families database. Nucleic Acids Res 2008, 36:D28I-288.
Publish with Bio Med Central and every scientist can read your work free of charge

"BioMed Central will be the most significant development for disseminating the results of biomedical research in our lifetime. "

Sir Paul Nurse, Cancer Research UK

Your research papers will be:

- available free of charge to the entire biomedical community

- peer reviewed and published immediately upon acceptance

- cited in PubMed and archived on PubMed Central

- yours - you keep the copyright
BioMedcentral 